\title{
Enhancement of Visual Contrast in Fluorescence Endoscopy
}

\author{
Thomas Stehle and Alexander Behrens and Til Aach \\ Institute of Imaging and Computer Vision \\ RWTH Aachen University, 52056 Aachen, Germany \\ tel: +49 24180 27860, fax: +49 2418022200 \\ web: www.lfb.rwth-aachen.de
}

in: Proceedings of the IEEE International Conference on Multimedia and Expo. See also $\mathrm{BIBT}_{\mathrm{E}} \mathrm{X}$ entry below.

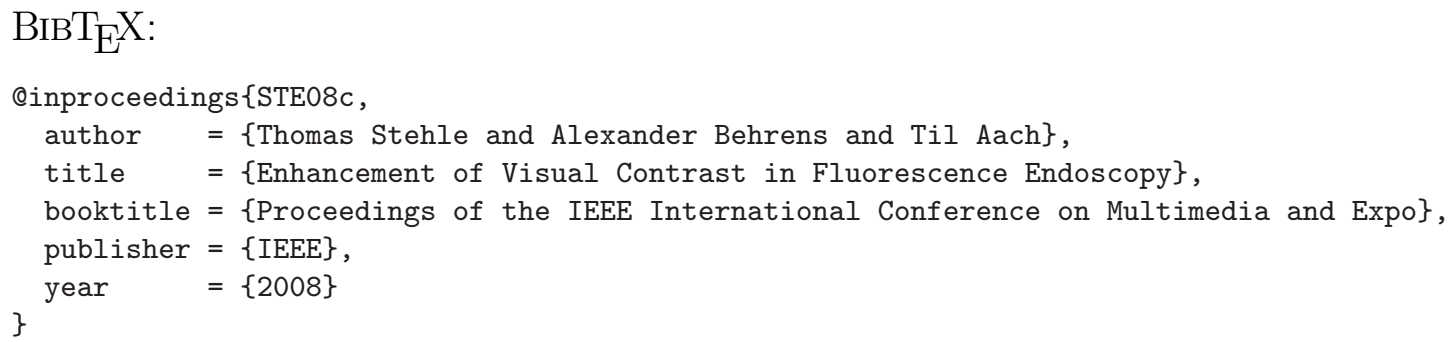

(C) 2008 IEEE. Personal use of this material is permitted. However, permission to reprint/republish this material for advertising or promotional purposes or for creating new collective works for resale or redistribution to servers or lists, or to reuse any copyrighted component of this work in other works must be obtained from the IEEE. 


\title{
ENHANCEMENT OF VISUAL CONTRAST IN FLUORESCENCE ENDOSCOPY
}

\author{
Thomas Stehle, Alexander Behrens, and Til Aach \\ Institute of Imaging \& Computer Vision, RWTH Aachen University, D-52056 Aachen, Germany
}

\begin{abstract}
Bladder tumors are difficult to recognize during an endoscopy of the bladder (cystoscopy) using a conventional white light source. Therefore, a technique called photo dynamic diagnosis (PDD) or fluorescence endoscopy is used to enhance both sensitivity and specificity of tumor detection. As preparation for a PDD cystoscopy, a marker substance is brought into the patient's bladder which leads to a fluorescence of cancerous tissue if a suitable light source is used. Even though tumors are better distinguishable from healthy bladder tissue, the contrast between both types of tissue can still be low and it further decreases with the decay of the marker substance.

To overcome these difficulties, we enhance the contrast between fluorescence and background. To this end, we perform a color space transformation which separates fluorescence information from the remaining information. In this new color space, we apply a non-linear transformation to the fluorescence component which leads to a better separation of fluorescing and non-fluorescing pixels. Finally, the image is transferred back to the RGB color space. For clinical evaluation, we implemented the proposed algorithm as plug-in for the real-time capable software framework RealTimeFrame.
\end{abstract}

Index Terms - Bladder, endoscopy, cystoscopy, fluorescence, photo dynamic diagnosis, PDD

\section{INTRODUCTION}

Cancer of the bladder has its highest incidence in industrialized countries. The greatest risk factors are smoking, contact with aromatic amines (e.g. through working in the dye industry), bilharziosis, and age. According to the National Institutes of Health (NIH) approximately 67,000 people were diagnosed with the disease and the number of deaths is 13,750 in 2007 in the United States.

Cancer of the bladder can be diagnosed and treated during an endoscopy of the bladder (so called cystoscopy). A cystoscope is brought through the urethra into the bladder, which is filled with isotonic saline solution. The cancerous tissue can then be removed using endoscopic tools, e. g. a resectoscope cutting loop.

Tumors in advanced stages are characterized by large

We would like to thank Olympus Winter \& Ibe GmbH, Hamburg, Germany for funding this project.
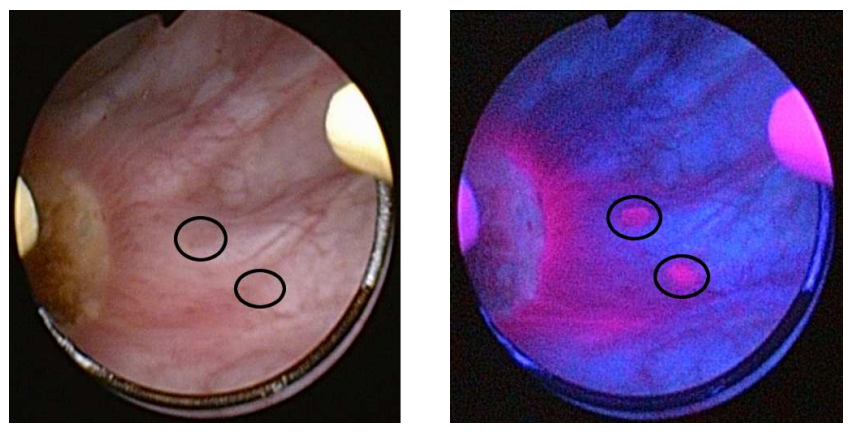

(a) Two papillary tumors
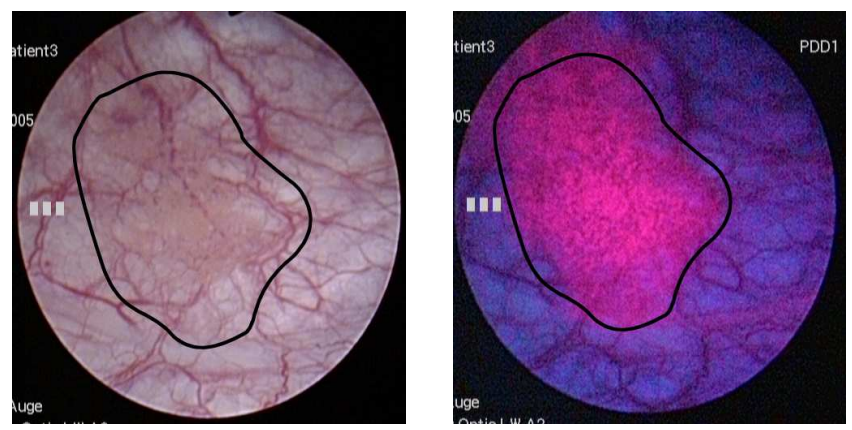

(b) A flat tumor

Fig. 1. Example of two types of tumors in normal (left) and PDD (right) illumination.

vesicular abscesses which can easily be detected because of their three dimensional morphology. Small vesicular tumors (see left hand side of Fig. 1a) as well as evolving flat tumors (see left hand side of Fig. 1b) in the bladder, however, are difficult to recognize because they do not differ strongly from the surrounding healthy tissue in terms of color or texture.

To overcome this difficulty, a technique called photo dynamic diagnosis (PDD) or fluorescence endoscopy can be used. A marker substance, like 5-aminolaevulinic acid (5ALA), is brought into the patient's bladder two and a half hours before the actual intervention. It accumulates at locations with high metabolic rate such as tumors. Under a special blue narrow band illumination 5-ALA starts fluorescing in red so healthy and cancerous tissues can be distinguished better (see right hand sides of Figs. 1a and 1b). Numerous studies $[1,2]$ indicate a higher sensitivity and specificity compared 
to normal white light cystoscopy.

During clinical collaboration with a fluorescence endoscopy specialist, the problem was stated that the contrast between fluorescing and non-fluorescing tissues can still be low and the boundaries of tumors can be hard to recognize. This problem increases progressively during the cystoscopy as 5-ALA decays in the fluorescence process which leads to a reduced contrast between bladder wall and tumor tissue. Therefore, we developed an algorithm which is suitable to enhance the contrast between both types of tissue in real-time $(720 \times 576$ pixels, 25 frames per second). This algorithm is presented in the following section.

\section{COLOR CONTRAST ENHANCEMENT}

Our approach aims at increasing contrast between two different colors, namely the non-fluorescing blue bladder wall and the fluorescing red tumor tissue. Whereas contrast (and its enhancement) is clearly defined in case of gray scale images, color contrast enhancement is not straight forward.

First, we present the derivation of a color space in which the fluorescence component is separated from the remaining color components and can therefore be enhanced in contrast. As always the same kind of light source is used and as the fluorescence always submits light of the same wavelength, we can assume the stationarity of both color classes. This implies that the color space has to be computed only once and not for every frame to enhance.

In a second step, we apply a non-linear transform on the fluorescence component which implements the actual contrast enhancement [3].

After the transformation back to RGB color space, the images can be displayed on a computer monitor. Fig. 2 gives an overview over the whole procedure.

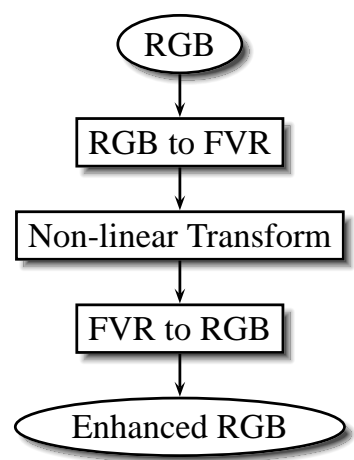

Fig. 2. Contrast enhancement overview.

\subsection{FVR Color Space}

In literature, there are many color spaces known, see e. g. [4] for an overview. All these color spaces separate information linearly or non-linearly to analyze or manipulate each component independently. In the following, we present the deriva- tion of a color space which optimally separates information in PDD endoscopy.

To analyze the distribution of the colors, we choose the representation of colors as vectors in the three dimensional RGB color space. Fig. 3 gives an impression of their distribution. As can be seen, the cluster is not aligned with any of the coordinate axes. Apparently, the largest variances of the cluster are in direction of the difference between fluorescing (red) and non-fluorescing (blue) colors, and in direction of brightness. There is only small variance perpendicular to both these axes. These are the desired axes of the new color space.

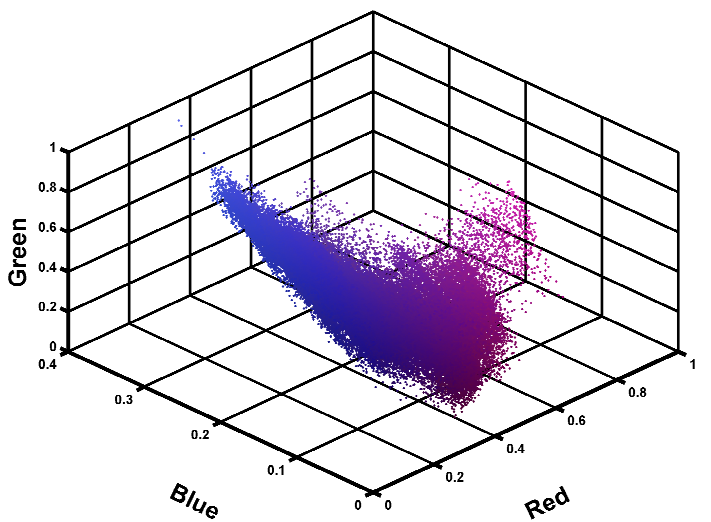

Fig. 3. Distribution of color vectors from a typical PDD endoscopy image in RGB color space.

A statistical measure of the spatial distribution is the covariance matrix

$$
C=\sum_{i}\left(\begin{array}{l}
R_{i}-\bar{R} \\
G_{i}-\bar{G} \\
B_{i}-\bar{B}
\end{array}\right) \cdot\left(\begin{array}{l}
R_{i}-\bar{R} \\
G_{i}-\bar{G} \\
B_{i}-\bar{B}
\end{array}\right)^{T}
$$

of color vectors from a typical PDD image where fluorescing and non-fluorescing pixels cover areas of approximately the same size. In Eq. (1), $\left(R_{i}, G_{i}, B_{i}\right)^{T}$ are the color vectors, $(\bar{R}, \bar{G}, \bar{B})^{T}$ is the cluster's mean color vector, and $(\cdot)^{T}$ means transposed.

The covariance matrix holds information of an ellipsoid whose axes describe the color cluster's extents, i.e. the directions of the largest variances which are the sought axes [5]. As the covariance matrix is a positive semi-definite matrix, we can use the principal component analysis (PCA) to find these axes which are then chosen as base vectors of the FVR color space.

The PCA gives two large and one small eigenvalues. The eigenvector corresponding to the first large eigenvalue describes the axis associated with fluorescence information and is therefore called Fluorescence axis. Fig. $4 \mathrm{~b}$ shows the fluorescence channel in which two vesicular tumors are clearly visible. The eigenvector corresponding to the second large eigenvalue describes the axis associated with brightness information. As it provides a good visualization of blood vessels, 
it is called Vascularization axis (see Fig. 4c). The eigenvector corresponding to the small eigenvalue does not contain much information and is therefore called Residual axis (see Fig. 4d).

Exemplary normalized eigenvalues for the eigenvectors defining the fluorescence, vascularization, and residual axes are $1,0.34$, and 0.02 , respectively. This confirms our observation of the color cluster's variances. Depending on the image data, however, the largest variance can either be caused by color change or by brightness change. If a different region of interest (ROI) in the same frame is chosen for the computation of the covariance matrix, normalized eigenvalues for $\mathrm{F}, \mathrm{V}$, and $\mathrm{R}$ like $0.4,1$, and 0.02 can occur. Therefore, the eigenvalues cannot be used to distinguish reliably between fluorescence or vascularisation axis. Instead, we compare the absolute values of the first entries of the eigenvectors (weights for the red color component). The eigenvector with the largest red component is defined as fluorescence axis as this is the dominant color in fluorescence.

The transform to the FVR color space can now be written as

$$
\left(\begin{array}{l}
F \\
V \\
R
\end{array}\right)=\left(\begin{array}{ccc}
- & \vec{b}_{1}^{\mathrm{T}} & - \\
- & \vec{b}_{2}^{\mathrm{T}} & - \\
- & \vec{b}_{3}^{\mathrm{T}} & -
\end{array}\right) \cdot\left(\begin{array}{l}
R-\bar{R} \\
G-\bar{G} \\
B-\bar{B}
\end{array}\right)
$$

where the $\vec{b}_{i}^{\mathrm{T}}$ correspond to the respective base vectors in row orientation.

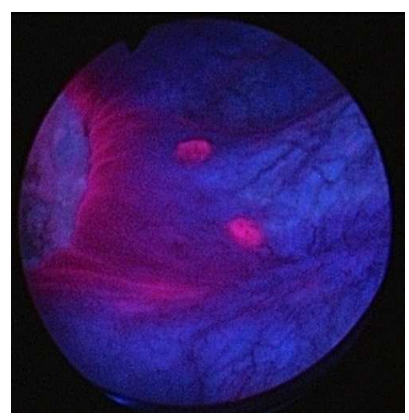

(a) Original representation

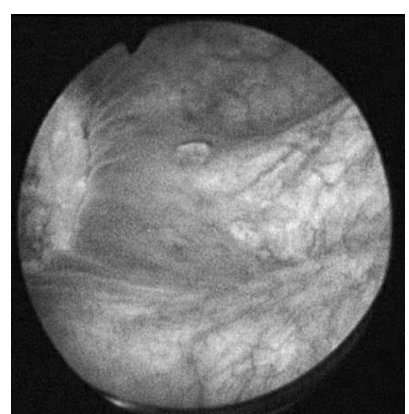

(c) Vascularization channel

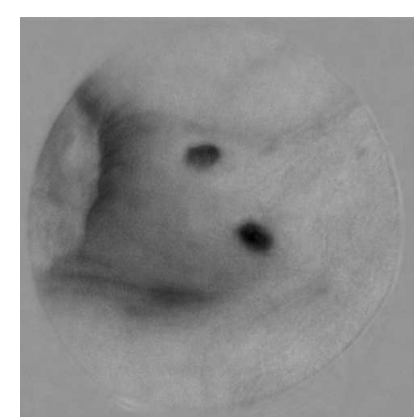

(b) Fluorescence channel

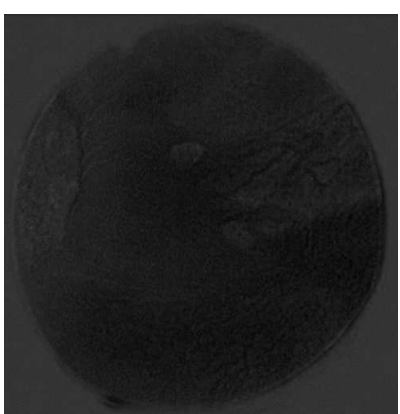

(d) Residual channel
Fig. 4. Information in the different channels of FVR color space.
Fig. 5 shows the distribution of color vectors in the FVR color space. The color vectors are decorrelated and aligned to the color space axes which can now be interpreted semantically.

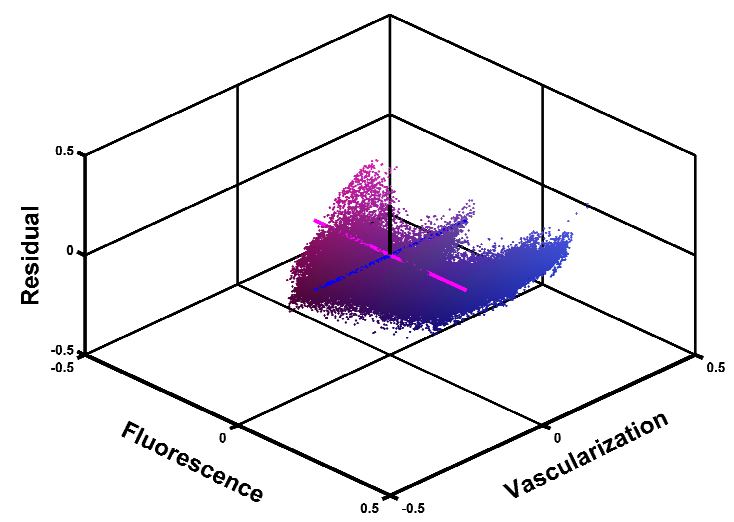

Fig. 5. Distribution of color vectors from a typical PDD endoscopy image in FVR color space. The color space axes $\mathrm{F}$ (magenta), V (blue), and R (black) have been added for illustrative purpose.

\subsection{Non-linear Contrast Enhancement}

As can be seen in Fig. 5, there is a smooth transition between fluorescing and non-fluorescing color vectors. To enhance the contrast between both classes, we separate the vectors which are located on the positive side of the fluorescence axis from the ones located on the negative side. Earlier clinical collaborations imply that the influence on the image should be as large as necessary but also as small as possible [6]. Therefore, color vectors, which are located far from the origin, and already exhibit a good contrast to the other class of color vectors, are not modified.

These properties are achieved by the application of the non-linear transformation

$$
n(F)=F \cdot\left(1+\alpha \cdot e^{-F^{2} / \beta}\right)
$$

to the fluorescence component of the color vectors. The constants $\alpha$ and $\beta$ are used to adjust the width of the non-linear influence and the function's slope in the origin. These parameters can be adapted during the endoscopy to compensate for the decay of 5-ALA. Fig. 6 depicts an example of such a non-linear function.

\section{EVALUATION}

Fig. 7 shows the effect of the proposed algorithm. On the left hand side, the original image is shown. The right hand side shows the output of our algorithm. The fluorescence of the bladder wall is reduced while the tumor's fluorescence is 


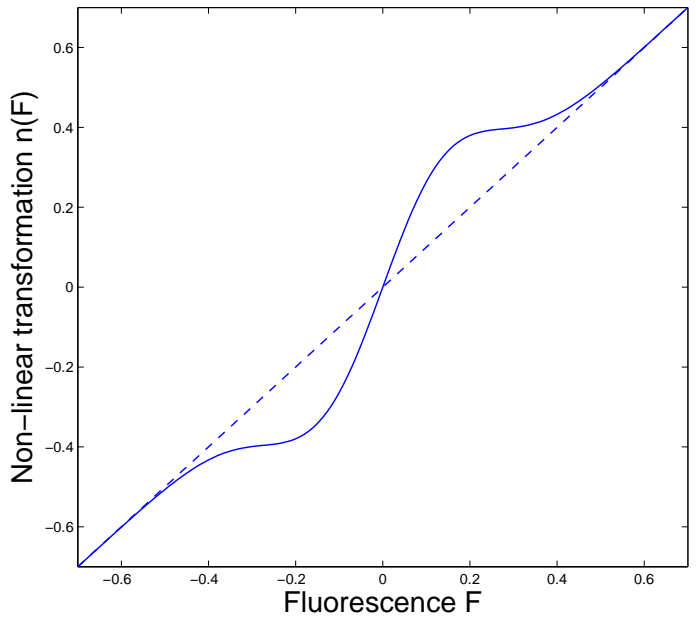

Fig. 6. Solid: Non-linear transformation for contrast enhancement in the fluorescence channel. $(\alpha=2.1, \beta=0.05)$ Dashed: Identity transformation. Only values near to the origin are affected by the non-linear transformation. Values far from the origin remain identical.

increased. This leads to a better contrast between both types of tissue.
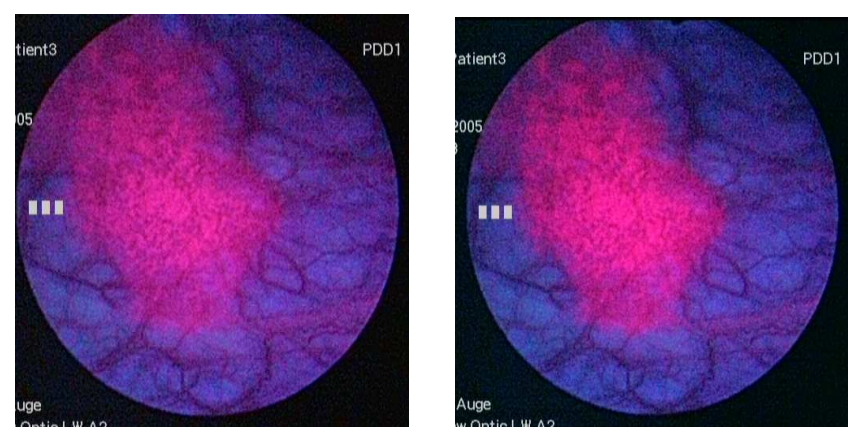

Fig. 7. Example of contrast enhancement algorithm. Left: Original image. Right: Image after proposed contrast enhancement. Fluorescence is reduced in the background but not in the tumor.

So far, we have achieved promising results which we plan to evaluate with our clinical partner. Therefore, we developed a real-time capable demonstrator platform which consists of high-performance standard PC components: $2 \times$ Intel Xeon 5140 dual core processors with $2.33 \mathrm{GHz}$, a GeForce 7950 GX2 video adapter, and 2 GB RAM. As software framework we developed RealTimeFrame [7], which provides a CPU efficient framework for real-time video processing. Because of the modular structure of RealTimeFrame, our method could easily be implemented as plug-in for the framework.

\section{SUMMARY}

Being directly motivated by the feedback of a physician, we developed an approach to enhance color contrast in fluorescence endoscopy. As first step, we defined a new color space which separates fluorescence information from the remaining signal using the principal component analysis. Given this color space, we developed a non-linear transformation which increases contrast between fluorescing and non-fluorescing tissue while it preserves already strong contrasts. Finally, for clinical evaluation, we developed a demonstrator platform and implemented the proposed algorithm as plug-in for the real-time capable video processing framework RealTimeFrame.

\section{ACKNOWLEDGEMENTS}

We would like to thank Dr. med. Andreas Auge, Waldklinikum Gera, Germany for sharing his opinion and giving feedback on this project.

\section{REFERENCES}

[1] Claus R. Riedl, Dmitri Daniltchenko, Frank Koenig, Reiner Simak, Stefan A. Loening, and H. Pflueger, "Fluorescence endoscopy with 5-aminolevulinic acid reduces early recurrence rate in superficial bladder cancer," The Journal of Urology, vol. 165, no. 4, pp. 1121-1123, Apr. 2001.

[2] Edwin Hungerhuber, Herbert Stepp, Martin Kriegmair, Christian Stief, Alfons Hofstetter, Arndt Hartmann, Ruth Knuechel, Alexander Karl, Stefan Tritschler, and Dirk Zaak, "Seven years' experience with 5-aminolevulinic acid in detection of transitional cell carcinoma of the bladder," Urology, vol. 69, no. 2, pp. 260-264, Feb. 2007.

[3] Thomas Stehle, Alexander Behrens, Matthias Bolz, and Til Aach, "Visual enhancement of fascial tissue in endoscopy," in SPIE Medical Imaging, San Diego, 2008.

[4] Konstantinos Plataniotis and Anastasios Venetsanopoulos, Color Image Processing and Applications, Springer, 2000.

[5] J. Tan, T.S.C.; Kittler, "Colour texture analysis using colour histogram," Vision, Image and Signal Processing, IEE Proceedings -, vol. 141, no. 6, pp. 403-412, 1994.

[6] Martin Stahl, Til Aach, and Sabine Dippel, "Digital radiography enhancement by nonlinear multiscale processing," Medical Physics, vol. 27, no. 1, pp. 56-65, 2000.

[7] Sebastian Gross, Thomas Stehle, Alexander Behrens, and Til Aach, "RealTimeFrame - a real time image processing solution for medical environments," in 41 . DGBMT-Jahrestagung Biomedizinische Technik (BMT 2007), Aachen, September 26-29 2007. 